\title{
Vortex-Glass Superconductivity: A Possible New Phase in Bulk High-T $\boldsymbol{c}_{c}$ Oxides
}

\author{
Matthew P. A. Fisher \\ IBM Research Division, T. J. Watson Center, Yorktown Heights, New York 10598
}

(Received 31 October 1988)

\begin{abstract}
The possibility of a new thermodynamic phase in the mixed state of bulk, disordered, type-II superconductors is suggested: a vortex-glass superconductor. This phase lacks conventional off-diagonal long-ranged order, yet is argued to be a true superconductor with vanishing dc resistance. In this phase metastable currents are predicted to decay as $(\ln t)^{-1 / \mu}$, with $\mu(\leq 1)$ a universal exponent. Relevance to experiments on bulk high- $T_{c}$ oxides is mentioned.
\end{abstract}

PACS numbers: $74.60 . \mathrm{Ge}, 74.60 . \mathrm{Ec}$

It has long been recognized that disorder plays a critical role in the static and dynamic properties of the mixed state in type-II superconductors. Disorder pins the vortex lines leading to an unobservably low voltage (creep rate) at small currents ${ }^{1}$ and destroys the translational long-ranged order of the Abrikosov flux lattice. ${ }^{2}$

Effects of disorder appear to be particularly acute in the new high- $T_{c}$ oxides. Significant hysteresis and irreversibility is observed in the magnetic response of the mixed state in $\mathrm{Ba}-\mathrm{La}-\mathrm{Cu}-\mathrm{O}$ powders ${ }^{3}$ and $\mathrm{Y}-\mathrm{Ba}-\mathrm{Cu}-\mathrm{O}$ crystals, ${ }^{4}$ even at high temperatures comparable to $T_{c}$. Moreover, Bitter decorations on some $\mathrm{YBaCuO}$ crystals show only short-range translational order $(\sim 2-3$ lattice spacing) in the flux lattice. ${ }^{5}$

Since disorder destroys the translational long-ranged order of the flux lattice, ${ }^{2}$ a natural question arises. Is there a sharp equilibrium phase boundary separating the normal phase at high temperatures and fields, from the "flux creep phase" at low $T$ and $H \gtrsim H_{c 1}$ ? The original Anderson-Kim theory, ' although predicting a strong crossover from fast to slow dynamics upon cooling, gives no sharp phase boundary since, even at low $T$, a nonzero (flux flow) resistance is predicted, as in the normal phase. The purpose of this Letter is to argue that in bulk disordered systems (but not thin films) there does, in fact, exist a sharp equilibrium phase boundary. Below this boundary there exists a new thermodynamic phase, which I refer to as a vortex-glass superconductor (see Fig. 1).

Glass phases in dirty superconductors have been suggested previously in numerical simulations on Josephson-junction-array models, ${ }^{6}$ and within a mean-field treatment of a granular superconductor. ${ }^{7}$ In the latter, a glass phase, similar to an $X Y$ spin-glass, was found when approximately one magnetic flux quantum penetrated a typical plaquette. Studies on $X Y$ spin-glasses, ${ }^{8}$ however, indicate that a true glass phase does not exist below $D=4$, and so caution is necessary. Moreover, it is not immediately clear what relevance the above models have for single-crystal superconductors with microscopic disorder. I consider a model in the continuum with microscopic pinning, and by analyzing a low-dimensional generalization, find strong evidence for the existence of a true vortex-glass phase in $3 \mathrm{D}$ (but not $2 \mathrm{D}$ ). This is not inconsistent with the 3D $X Y$ spin-glass results, since an applied magnetic field leads to considerably less frustration: All plaquettes which lie in planes with normals perpendicular to the field have no threading flux and are unfrustrated. As in Ref. 7, the vortex-glass phase is characterized by a nonzero Edwards-Anderson order parameter, yet does not have conventional off-diagonal long-ranged order (ODLRO). It is argued herein, though, to be a true superconductor with zero dc resistance.

Consider the mixed state of an extreme type-II superconductor (Ginzburg-Landau parameter $\kappa=\lambda / \xi \gg 1$ ) described by a standard Ginzburg-Landau Hamiltonian

$$
\begin{array}{r}
\mathscr{H}=\int d^{3} R\left(\frac{1}{2 m}|(\nabla-i e \mathbf{A}) \psi|^{2}+f(\psi)\right. \\
\left.+\frac{1}{8 \pi} \mathbf{B}^{2}-\frac{1}{4 \pi} \mathbf{H} \cdot \mathbf{B}\right),
\end{array}
$$

with $f(\psi)=a(\mathbf{R})|\psi|^{2}+b|\psi|^{4}, \mathbf{B}=\nabla \times \mathbf{A}$, and $\mathbf{H}=H \hat{\mathbf{z}}$ a constant applied field. Quenched disorder is included in the $|\psi|^{2}$ term. The partition function is $Z$ $=\operatorname{Tr}_{\psi, A} \exp (-\beta \mathcal{H})$ with a trace over both $\psi$ and the gauge field $\mathbf{A}$.

For temperatures below $T_{c}$ and fields above $H_{c 1}$, the important degrees of freedom are the vortex lines threading the sample. Indeed, there exists a dual representation ${ }^{9}$ of a lattice version of (1) in terms of $2 \mathrm{D}$ bosons at $T=0$, with the boson world lines $\mathbf{r}_{i}(\tau)$, parametrized by time, corresponding to the vortex lines $\mathbf{r}_{i}(z)=\left(x_{i}(z)\right.$, $\left.y_{i}(z)\right)$, parametrized by $z$. This dual representation allows a study of disorder on the mixed state, by applying ideas and techniques from the problem of disordered superfluids. ${ }^{10}$ In the continuum the appropriate second quantized boson Hamiltonian is $\hat{\mathscr{H}}=\hat{\mathscr{H}}_{0}+\hat{\mathscr{H}}_{1}+\hat{\mathscr{H}}_{p}$ with

$$
\begin{aligned}
& \hat{\mathscr{H}}_{0}=\int_{r}\left(\frac{-T^{2}}{2 \epsilon_{1}} \hat{\Phi}^{\dagger}(r) \nabla_{r}^{2} \hat{\Phi}(r)+\frac{1}{4 \pi}\left(H_{c 1}-H\right) \hat{B}(r)\right), \\
& \hat{\mathscr{H}}_{1}=\frac{1}{2}\left(8 \pi^{2} \lambda^{2}\right)^{-1} \int_{r, r^{\prime}} \hat{B}(r) K_{0}\left(\frac{r-r^{\prime}}{\lambda}\right) \hat{B}\left(r^{\prime}\right), \\
& \hat{\mathscr{H}}_{p}=\int d^{2} r V_{p}(\mathbf{r}, z) \hat{B}(r),
\end{aligned}
$$


and $Z=\operatorname{Tr} T_{z}\left\{\exp \left[-T^{-1} \int d z \hat{\mathscr{H}}(z)\right]\right\}$, with $T_{z}$ an "imaginary time" ordered product. Here $\hat{\Phi}(r)$ is a boson field operator, and $\hat{B}(r) \equiv \phi_{0} \hat{\Phi}^{\dagger}(r) \hat{\Phi}(r) \quad\left(\phi_{0}=h c / 2 e\right)$ is proportional to the vortex-line (and boson) density, i.e., is the local magnetic field $B_{z}$. The modified Bessel function $K_{0}(x) \approx(\pi / 2 x)^{1 / 2} e^{-x}$ (for $x$ large) describes the interaction between vortex lines (bosons), $\epsilon_{1} \equiv H_{c_{1}} \phi_{0} / 4 \pi$ is the vortex-line tension (boson mass), and $V_{p}(\mathbf{r}, z)$ is a random quenching pinning potential. Note that $V_{p}$ corresponds to a space and "time" dependent potential for the 2D bosons. For simplicity we take Gaussian disorder, uncorrelated in space and "time," with mean zero and variance $\Delta$, appropriate for microscopic pinning centers (e.g., oxygen defects).

There are (at least) three important energy scales in (2). The pinning energy for a microscopic impurity is a fraction $p$ of the condensation energy ${ }^{1,11} H_{c}^{2} \xi^{2}$, i.e., $\sim p H_{c 1} \phi_{0}$. [Therefore $\Delta \sim\left(p H_{c 1}\right)^{2} n_{p}$, where $n_{p}$ is the density of pinning centers.] Normally ${ }^{11,12} p \sim 10^{-3}$, but in the high- $T_{c}$ oxides with $\xi$ comparable to the atomic scale one expects $p \sim 1$. This energy is then roughly comparable to the (interaction energy)/length between vortices separated by $\lambda$ (or less): $\phi_{0}^{2} / \lambda^{2} \sim H_{c 1} \phi_{0}$. The entropic contribution per length to the free energy of a vortex line confined by its neighbors to a region of, say, $\lambda^{2}$, is roughly $T^{2} / \epsilon_{1} \lambda^{2} \sim T^{2} / \phi_{0}^{2}$ (i.e., the boson kinetic energy $\left.\hbar^{2} / m \lambda^{2}\right)$. Thus as emphasized by Nelson, ${ }^{9}$ high- $T_{c}$ oxides with large $T_{c}$ 's and modest $H_{c}$ 's are likely to be affected by entropic effects well below $T_{c}$.

The Meissner phase, $\langle B(r)\rangle=0$, corresponds to an absence of vortex lines (bosons) in (2), i.e., $H<H_{c 1}$. For $H>H_{c 1}$ an Imry-Ma ${ }^{12}$ argument indicates that pinning destroys the translational (and presumably orientational) order of the flux lattice since, at large scales $L \gtrsim L_{c}$, fluctuations in the bulk pinning energy $\left(\sim \Delta^{1 / 2} L^{3 / 2}\right)$ always dominate the crystal elastic energy $\left(\sim H_{c 1} L^{3} / L^{2}\right)$. Equating these two energies gives $L_{c} \sim\left(n_{p} p^{2}\right)^{-1}$. Thus, consistent with experiment, ${ }^{5}$ the flux lattice in high- $T_{c}$ oxides $(p \sim 1)$ should typically be more disordered than in normal superconductors $\left(p \sim 10^{-3}\right)$.

For $H>H_{c 1}$ there is a competition between thermal fluctuations, which enhance vortex-line density fluctuations, and the pinning disorder and repulsive intervortex interactions which tend to minimize such fluctuations. At high temperatures thermal fluctuations will dominate and should result in an unpinned vortex-liquid phase. This phase (which is dual to the boson superfluid phase) has no ODLRO ${ }^{10}$ due to the thermal motion of the vortex lines. As suggested in Ref. 13, the vortex-liquid phase provides a particularly apt description of high $-T_{c}$ oxides in the temperature range below the mean-field $H_{c 2}$ line (where $\psi$ first attains a non-negligible amplitude and a description in terms of vortex lines is appropriate), yet, due to large fluctuations, above the Abrikosov phase or the "irreversibility" line where the flux lines are effectively pinned. Below we argue that this pinned

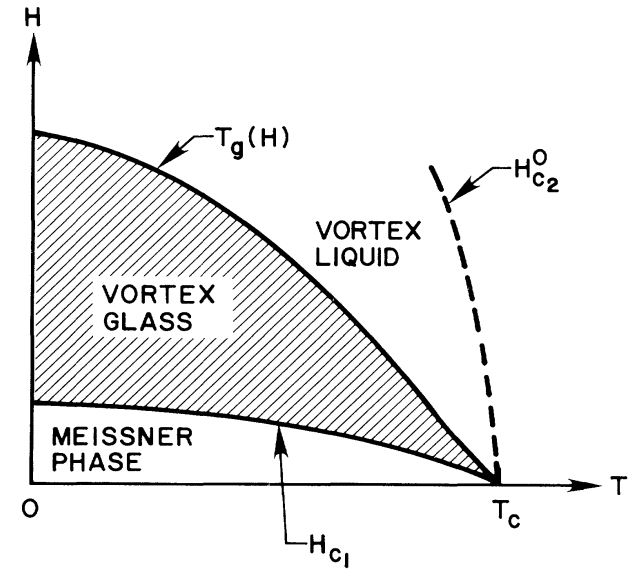

FIG. 1. Schematic phase diagram for a bulk dirty superconductor. The presence of a vortex-liquid regime, between the (mean-field) $H_{c 2}$ line and the vortex-glass phase, requires large fluctuations.

phase is really a vortex glass (see Fig. 1).

At low temperatures pinning disorder and interactions should dominate thermal effects. To search for a vortex-glass phase in this regime, consider ensemble averaging in (2). After replacing and employing a pathintegral representation of $Z$, the ensemble average over $V_{p}$ can be performed, yielding an effective replicated boson Hamiltonian ${ }^{14}$

$$
\hat{\mathscr{H}}_{n}=\sum_{\alpha=1}^{n}\left\{\hat{\mathscr{H}}_{0, \alpha}+\hat{\mathscr{H}}_{1, \alpha}\right\}-\frac{\Delta}{T} \int_{r} \sum_{\alpha, \beta} \hat{B}_{\alpha}(r) \hat{B}_{\beta}(r),
$$

with $\alpha, \beta$ replica indices and the $n \rightarrow 0$ limit understood. Equation (3) described $n$ species of vortex lines (bosons) interacting by an attractive interreplica interaction.

A vortex-glass instability is evident if one considers $n$ a positive integer greater than one. In the dilute limit, bosons in different replicas should bind together to form an $n$-molecule bound state. ${ }^{14}$ Since the dual Bose system is at $T=0$, these molecules should undergo an $n$-boson condensation, indicative of a disorder-dominated vortexglass instability. In thin films, this instability will be absent, since the inverse film thickness corresponds to temperature in the dual boson problem ${ }^{9}$ and thermal fluctuations will "ionize" the $n$ molecules. It remains to show that this instability survives the $n \rightarrow 0$ limit.

To see this we consider a low-dimensional generalization of (2), corresponding to vortex lines $x_{i}(z)$ restricted to lie in the $x-z$ plane. This model can be studied by employing methods introduced by Haldane ${ }^{15}$ to describe 1D boson systems. Haldane writes the Bose density operator as

$$
\hat{B}(x)=\left[B_{0}+\hat{\Pi}(x)\right] \sum_{m} \exp [\operatorname{im} \hat{A}(x)],
$$

where $B_{0}$ is the mean (vortex-line) density and $\Pi$ a small 
fluctuation. The choice $\partial_{x} A=B_{0}+\Pi \equiv B_{0}+\partial_{x} \tilde{A}$ captures the discrete nature of the boson field, since the sum in (4) is nonzero for integer $A / 2 \pi$ only. Thus the condition $A(x, z)=2 \pi l$ for $l$ integer specifies the location of the vortex lines. The partition function can be written as a functional integral over the field $\tilde{A}(x, z): Z$ $=\operatorname{Tr}_{\tilde{A}} \exp (-S)$. In the absence of disorder the effective action is ${ }^{15}$

$$
S_{0}=\frac{T_{g}}{8 \pi T} \int d x d z\left[v\left(\partial_{x} \tilde{A}\right)^{2}+v^{-1}\left(\partial_{z} \tilde{A}\right)^{2}\right],
$$

where $T_{g} v=\left(\partial B_{0} / \partial H\right)^{-1}$ and $T_{g} / v=H_{c 1} / B_{0}$. With disorder, after replicating and ensemble averaging a term proportional to $\Delta$ is generated, as in (3). Inserting $\hat{B}$ from (4) into (3) and retaining only the most important $(m= \pm 1)$ terms one has

$$
S_{p}=-\tilde{\Delta} \sum_{\alpha, \beta} \int_{x, z} \cos \left[\tilde{A}_{\alpha}(x, z)-\tilde{A}_{\beta}(x, z)\right],
$$

with $S_{n}=\Sigma_{\alpha} S_{0, \alpha}+S_{p}$. Here $\tilde{\Delta}=\Delta\left(B_{0} / T\right)^{2}$. The action (5) can be viewed as a $2 \mathrm{D}$ analog of the gauge theory (1). Indeed, the scalar field $A(x, z)$ plays the role of the vector potential, with $\mathbf{B} \sim\left(\partial_{z} A, \partial_{x} A\right)$.

The model (5) has been studied by Cardy and Ostlund ${ }^{14,16}$ in the context of a $2 \mathrm{D}$ random-field $X Y$ model with all vortices excluded. A perturbative renormalization group in $\Delta$ about the fixed line described by $(5 \mathrm{a})$ can be performed, and the $n \rightarrow 0$ limit taken. The resulting flow equation to linear order is $\partial \Delta / \partial l=2\left(1-T / T_{g}\right) \Delta$. For $T>T_{g}$, the pinning disorder scales to zero at long length scales and the vortex-liquid phase described by (5a) results. For $T<T_{g}$, however, $\Delta$ grows under renormalization, driving the system into a new disorderdominated phase, which I call a vortex glass. Since thermodynamic phases are generally more stable in higher dimensions, this 2D result provides strong evidence for the existence of a vortex-glass phase in bulk systems.

When $\Delta$ grows in $(5 \mathrm{~b})$, replicas $\tilde{A}_{\alpha}$ and $\tilde{A}_{\beta}$ tend to lock, suggesting that a Higgs-type mass term $\left(\tilde{A}_{\alpha}-\tilde{A}_{\beta}\right)^{2}$ is generated. An analogous term would be generated in (1) were the expectation value $q_{\mathrm{EA}} \equiv\left\langle\tilde{\psi}_{\alpha}^{*} \tilde{\psi}_{\beta}\right\rangle$ nonzero [where $\tilde{\psi}(x)=\psi(x) \exp \left[\int{ }^{x} \boldsymbol{i} \mathbf{A} \cdot d \boldsymbol{l}\right]$ is a gauge-invariant order parameter. Thus in the vortex-glass phase, although there is no ODLRO I expect there is a nonzero Edwards-Anderson order parameter (with $V$ the volume)

$$
q_{\mathrm{EA}}=\frac{1}{V} \int_{x}|\langle\tilde{\psi}(x)\rangle|^{2} \neq 0 .
$$

To study the dynamics of the vortex-glass phase, it is convenient to reinterpret the symmetry breaking (6) in terms of the vortex lines. Note that the action (5) is invariant under $A_{\alpha} \rightarrow A_{\alpha}+2 \pi N$, for integer $N$. Under this transformation, any given vortex line, specified say by the condition $A(x, z)=2 \pi l$, is transferred over and takes the place of the vortex line specified originally by $A(x, z)$ $=2 \pi(l+N)$. In other words, $A_{\alpha} \rightarrow A_{\alpha}+2 \pi N$ corre- sponds to a global center-of-mass shift by $N$ in the $\alpha$ th replica, arriving at a configuration which, up to relabeling of vortex lines, is identical to the original one. It is this symmetry which is broken in the vortex-glass phase. The vortex center of mass is thus effectively pinned by the disorder. Although only one pure glass phase is expected $^{17}$ in (5), it is convenient to treat the equivalent but shifted (or relabeled) phases as distinct and labeled by $N$. Symmetry breaking then implies that an infinite free-energy barrier separates each "phase" $N$ from its neighbors $N \pm 1$. A (Lorentz) force which acts to increase $N$, will tend to create drops of the $N+1$ phase in the metastable $N$ phase. As pointed out by Huse, ${ }^{18}$ the perimeter of such a drop can conveniently be viewed as a directed vortex loop, superimposed on the reference phase $N$. The loop will have an energy which grows without bound as its size $L \rightarrow \infty$.

In the physically relevant $3 \mathrm{D}$ case a current $J$, which produces a Lorentz force on the vortex lines, will tend to nucleate such vortex loops leading, in the steady state, to a finite flux-flow velocity $v$. Following arguments in Ref. 19 , one expects $v \sim \exp \left(-E_{c} / T\right)$, where $E_{c}$ is the energy of a critical nucleating vortex loop. Assuming that vortex-loop formation of size $L$ typically requires passing over energy barriers which (at $J=0$ ) scale as a positive power ${ }^{20}(\leq 1)$ of $L$ implies $E_{c} \sim(1 / J)^{\mu}$, with $\mu(\leq 1)$ a universal exponent characterizing the $T=0$ vortex-glass fixed point. ${ }^{20,21}$ Thus a nonlinear voltage response is expected:

$$
V \sim \exp \left[-\left(J_{c} / J\right)^{\mu}\right]
$$

Equation (7) indicates that the linear dc resistance will vanish. The vortex-glass phase is a true superconductor. ${ }^{22}$ Since a metastable current (or magnetization $\delta M$ ) will relax as $\partial J / \partial t \sim V$, integration of (7) implies $J(t)$ $\sim \delta M(t) \sim(\ln t)^{-1 / \mu}$ as $t \rightarrow \infty$.

ac magnetic-susceptibility measurements on bulk Y$\mathrm{Ba}-\mathrm{Cu}-\mathrm{O}$ crystals, ${ }^{4}$ in the presence of a dc field, indicate a set of frequency-dependent "irreversibility" lines $T_{\text {irr }}(H)$. For $T<T_{\text {irr }}$ flux lines have insufficient time to penetrate the sample in the period of the ac field. A collapse onto a nonzero curve $T_{g}(H) \equiv T_{\text {irr }}(H ; \omega \rightarrow 0)$ in the $\omega \rightarrow 0$ limit would be indicative of a true vortex-glass superconductor for $T<T_{g}$. [In the flux-creep picture $T_{\text {irr }}(H, \omega) \rightarrow 0$ as $\omega \rightarrow 0$.] For $T>T_{g}$, one would then expect, from scaling arguments, ${ }^{21} \quad T_{\text {irr }}(\omega)-T_{g}$ $\sim \omega^{1 /(z-1)^{v}}$ for small $\omega$, with $z$ the dynamical and $v$ the correlation-length critical exponents, respectively, associated with the vortex-liquid to vortex-glass transition.

Many experimentally relevant quantities, such as the critical behavior of the vortex-glass transition and finitefrequency response functions in the glass phase remain as theoretical challenges.

I thank D. S. Fisher and D. Huse for a critical reading of the manuscript and numerous clarifying conversations, and D. H. Lee, A. Malozemoff, and J. Toner for 
useful discussions.

'P. W. Anderson and Y. B. Kim, Rev. Mod. Phys. 36, 39 (1964).

${ }^{2}$ A. I. Larkin, Zh. Eksp. Teor. Fiz. 58, 1466 (1970) [Sov. Phys. JETP 31, 784 (1970)]; A. I. Larkin and Yu. N. Ovchinnikov, J. Low Temp. Phys. 34, 409 (1979).

${ }^{3}$ K. A. Müller, M. Takashige, and J. G. Bednorz, Phys. Rev. Lett. 58, 1143 (1987).

${ }^{4}$ A. P. Malozemoff, T. K. Worthington, Y. Yeshurun, and F. Holtzberg, Phys. Rev. B 38, 7203 (1988).

${ }^{5}$ P. L. Gammel, D. J. Bishop, G. J. Dolan, J. R. Kwo, C. A. Murray, L. F. Schneemeyer, and J. V. Waszczak, Phys. Rev. Lett. 59, 2592 (1987).

${ }^{6}$ C. Ebner and D. Stroud, Phys. Rev. B 31, 165 (1985).

${ }^{7}$ S. John and T. Lubensky, Phys. Rev. B 34, 4815 (1986).

${ }^{8}$ B. W. Morris, S. G. Colbourne, M. A. Moore, A. J. Bray, and J. Canisius, J. Phys. C 19, 1157 (1986).

${ }^{9}$ D. R. Nelson, Phys. Rev. Lett. 60, 1973 (1988); M. P. A. Fisher and D. H. Lee, Phys. Rev. B 39, 2756 (1989).

${ }^{10}$ D. S. Fisher and M. P. A. Fisher, Phys. Rev. Lett. 61, 1847 (1988).
${ }^{11} \mathrm{M}$. Tinkham, Introduction to Superconductivity (Krieger, Malabar, 1975).

${ }^{12}$ Y. Imry and S. K. Ma, Phys. Rev. Lett. 35, 1399 (1975).

${ }^{13}$ P. L. Gammel, L. F. Schneemeyer, J. V. Waszczak, and D. J. Bishop, Phys. Rev. Lett. 61, 1666 (1988).

${ }^{14}$ See M. Kardar, Nucl. Phys. B290 [FS20], 582 (1987), and references therein, where the related problem of directed walks in a random environment is discussed, both with and without replicas.

${ }^{15}$ F. D. M. Haldane, Phys. Rev. Lett. 47, 1840 (1981).

${ }^{16}$ J. L. Cardy and S. Ostlund, Phys. Rev. B 25, 6899 (1982).

${ }^{17}$ In the $3 \mathrm{D}$ case, a given pure state $\psi$ and all those related to it by a global phase rotation correspond to a single state in terms of vortex lines. The possibility of many pure states unrelated by symmetry is not considered here.

${ }^{18}$ D. A. Huse (private communication).

${ }^{19}$ J. S. Langer and M. E. Fisher, Phys. Rev. Lett. 19, 560 (1967).

${ }^{20}$ D. S. Fisher and D. A. Huse, Phys. Rev. B 38, 386 (1988).

${ }^{21}$ D. S. Fisher, D. Huse, and M. P. A. Fisher (unpublished).

${ }^{22}$ One can also argue that the superfluid density is nonzero in the vortex-glass phase. D. S. Fisher (private communication); and Ref. 21. 